\title{
Common Fixed Point Results in b-metric-like Spaces
}

\author{
Deepak Kumar \\ Department of Mathematics, G.M.N. (PG) College, Ambala Cantt, Haryana, India
}

\begin{abstract}
Fixed point theory is a powerful tool in mathematics. In this paper, we introduce a common fixed point results in new generalize b-metric-like spaces. This result extend and generalize many existing results in the literature.
\end{abstract}

Keywords: fixed point

\section{Introduction}

The concept of b-metric space was introduced and studied by czerwik [3]. since then serval papers have been dealt with fixed point theory for single-valued and multivalued operators in b-metric spaces. Amini-Harandi [2] introduced the notion of metric-like space, which is an interesting generalization of partial metric space. Recently, Mohammed Ali Algamdi [1] introduced a new generalization of metriclike space and partial metric space is called a b-metric-like space and studied some fixed point theorem of b-metric-like space. These results improved some well-known results in the literature.

\section{Preliminaries}

In this section, we recall some of the metric spaces and mappings as follows:

Definition 2.1. [1] A b-metric-like on a non empty set $X$ is a function $\vartheta: X \times X \rightarrow[0,+\infty)$ such that for all $p, q, r \in X$ and a constant $\mathrm{K} \geq 1$ the following three conditions hold true

(D1) if $\vartheta(p, q)=0 \Rightarrow p=q$

(D2) $\vartheta(p, q)=\vartheta(q, p)$

(D3) $\vartheta(p, q) \leq K(\vartheta(p, r)+\vartheta(r, q))$

The pair $(\mathrm{X}, \vartheta)$ is called a b-metric-like space.

Example 2.2. [1] Let $X=[0,+\infty)$. Define the function $\vartheta$ : $X^{2} \rightarrow[0,+\infty)$ by $\vartheta(p, q)=(p+q)^{2}$.Then

$(X, \vartheta)$ is a $b-$ metric - like space with constant $K$

$$
\begin{aligned}
& =2 \text {. Clearly }(X, \vartheta) \text { is not } a b \\
& - \text { metric or }
\end{aligned}
$$

metric - like space.Indeed, for all $p, q, r \in X$

$\vartheta(p, q)=(p+q)^{2} \leq(p+r+r+q)^{2}$

$$
=(p+r)^{2}+(r+q)^{2}+2(p+r)(r
$$$$
+q)
$$

$\leq 2\left[(p+r)^{2}+(r+q)^{2}\right]$

$=2(\vartheta(p, r)+\vartheta(r, q))$

and so (D3)holds. Clearly, (D1) and (D2)hold.

Definition 2.3. An element $(a, b) \in X \times X$ is called a coupled fixed point of $T: X \times X \rightarrow X$ if $a=T$

$(a, b)$ and $S(b, a)=T(b, a)$.
Definition 2.4. An element $(a, b) \in X \times X$ is called a coupled Coincidence point $S, T: X \times X \rightarrow X$ if $S(a, b)=$ $T(a, b)$ and $S(b, a)=T(b, a)$.

\section{Example 2.5.}

Let $X=\Re$ and $S, T: X \times X \rightarrow X$ defined as $S(a, b)=a+b-a b+\sin (a+b)$ and $T(a, b)$

$$
=a+b+\cos (a+b)
$$

for all $a, b$

$\in X$.Then $\left(0, \frac{\pi}{4}\right)$ and $\left(\frac{\pi}{4}, 0\right)$ are coupled coincidence points of $S$ and $T$.

Definition 2.6. An element

$(a, b) \in X \times X$ is called a of $S, T: X \times X \rightarrow X$ if $a=S(a, b)=T(a, b)$ and $b=S(b, a)=T(b, a)$.

\section{Example 2.7.}

Let $X=\Re$ and $S, T: X \times X \rightarrow X$ defined as $S(a, b)=a b$ and $T(a, b)=a+(b-a)^{2}$

for all $a, b$

$\in X$.Then $(0,0)$ and $(1,1)$ are common coupled fixed points of $S$ and $T$.

\section{Main Results}

Theorem 3.1. Let $(X, \vartheta)$ be a complete $b-$ metric like slpace and a constant $K \geq 1$ and let the mapping $S, T: X \times X \rightarrow X$ satisfy $\vartheta(S(a, b), T(u, v))$

$$
\begin{aligned}
& \leq \alpha \frac{\vartheta(a, u)+\vartheta(b, u)}{2} \\
& +\beta \frac{\vartheta(a, S(a, b)) \vartheta(u, v))}{(1+\vartheta(a, u)+\vartheta(b, u))} \\
& +\gamma \frac{\vartheta(u, S(a, b)) \vartheta(a, T(u, v))}{(1+\vartheta(a, u)+\vartheta(b, u))}(3.1) \\
& \in X \text { and } \alpha, \beta \geq 0 \text { with } K \alpha+\beta \\
& <1 \text { and } \alpha+\gamma \\
& <1 . \text { Then } S \text { and } T \text { have a unique }
\end{aligned}
$$

for all $a, b, u, v \in X$ and $\alpha, \beta \geq 0$ with $K \alpha+\beta$

common coupled fixed point in $X$.

Proof. Step 1 : Firstly, We show that $a_{n}, b_{n}$ are Cauchy sequence in $\mathrm{X}$.

Let $a_{0}, b_{0} \in X$ be any arbitrary points. Define $a_{2 k+1}=$ $S\left(a_{2 k}, b_{2 k}\right), b_{2 k+1}=S\left(b_{2 k}, a_{2 k}\right)$ and

$a_{2 k+1}=T\left(a_{2 k+1}, b_{2 k+1}\right), b_{2 k+2}=T\left(b_{2 k+1}, a_{2 k+1}\right)$ for $k=0,1,2,3, \ldots \ldots$ 


\section{International Journal of Science and Research (IJSR) \\ ISSN (Online): 2319-7064}

Index Copernicus Value (2013): 6.14 | Impact Factor (2014): 5.611

Now

$\vartheta\left(a_{2 k+1}, a_{2 k+2}\right)=\vartheta\left(S\left(a_{2 k}, b_{2 k}\right), T\left(a_{2 k+1}, b_{2 k+1}\right)\right)$

$\vartheta\left(a_{2 k+1}, a_{2 k+2}\right) \leq \alpha \frac{\vartheta\left(a_{2 k}, a_{2 k+1}\right)+\vartheta\left(b_{2 k}, b_{2 k}, b_{2 k+1}\right)}{2}+$

$\beta \frac{\vartheta\left(a_{2 k}, S\left(a_{2 k}, b_{2 k}\right)\right) \vartheta\left(a_{2 k+1}, T\left(a_{2 k+1}, b_{2 k+1}\right)\right)}{\left(1+\vartheta\left(a_{2 k} a_{2 k+1}\right)+\vartheta\left(b_{2 k}, b_{2 k+1}\right)\right)}+$

$$
\gamma \frac{\vartheta\left(a_{2 k+1}, S\left(a_{2 k}, b_{2 k}\right)\right) \vartheta\left(a_{2 k}, T\left(a_{2 k+1}, b_{2 k+1}\right)\right)}{\left(1+\vartheta\left(a_{2 k}, a_{2 k+1}\right)+\vartheta\left(b_{2 k}, b_{2 k+1}\right)\right)} \vartheta
$$

$\left(a_{2 k+1}, a_{2 k+2}\right)$

$=\alpha \frac{\vartheta\left(a_{2 k}, a_{2 k+1}\right)+\vartheta\left(b_{2 k}, b_{2 k+1}\right)}{2}$

$+\beta \frac{\vartheta\left(a_{2 k}, a_{2 k+1}\right) \vartheta\left(a_{2 k+1}, a_{2 k+1}, a_{2 k+2}\right)}{\left(1+\vartheta\left(a_{2 k}, a_{2 k+1}\right)+\vartheta\left(b_{2 k}, b_{2 k+1}\right)\right)}+$

$\gamma \frac{\vartheta\left(a_{2 k+1}, a_{2 k+1}\right) \vartheta\left(a_{2 k}, a_{2 k+2}\right)}{\left(1+\vartheta\left(a_{2 k}, a_{2 k+1}\right)+\vartheta\left(b_{2 k}, b_{2 k+1}\right)\right)}$

$\vartheta\left(a_{2 k+1}, a_{2 k+2}\right) \leq \alpha \frac{\vartheta\left(a_{2 k}, a_{2 k+1}\right)+\vartheta\left(b_{2 k}, b_{2 k+1}\right)}{2}$

$+\beta \frac{\vartheta\left(a_{2 k}, a_{2 k+1}\right) \vartheta\left(a_{2 k+1}, a_{2 k+2}\right)}{\left(1+\vartheta\left(a_{2 k}, a_{2 k+1}\right)+\vartheta\left(b_{2 k}, b_{2 k+1}\right)\right)}$

$\gamma\left(2 \vartheta\left(a_{2 k+1}, a_{2 k+2}\right)\right)$

$\vartheta\left(a_{2 k+1}, a_{2 k+2}\right) \leq \alpha \frac{\vartheta\left(a_{2 k}, a_{2 k+1}\right)}{2}+\alpha \frac{\vartheta\left(b_{2 k}, b_{2 k+1}\right.}{2}$

$$
+\beta \vartheta\left(a_{2 k+1}, a_{2 k+2}\right)
$$$$
+\gamma\left(2 \vartheta\left(a_{2 k+1}, a_{2 k+2}\right)\right)
$$

$(1-\beta-2 \gamma) \vartheta\left(a_{2 k+1}, a_{2 k+2}\right)$

$$
\leq \alpha \frac{\vartheta\left(a_{2 k}, a_{2 k+1}\right)}{2}+\alpha \frac{\vartheta\left(b_{2 k}, b_{2 k+1}\right)}{2}
$$

$\vartheta\left(a_{2 k+1}, a_{2 k+2}\right) \leq \alpha \frac{\vartheta\left(a_{2 k}, a_{2 k+1}\right)}{2(1-\beta-2 \gamma)}+\alpha \frac{\vartheta\left(b_{2 k}, b_{2 k+1}\right)}{2(1-\beta-2 \gamma)}$

$\vartheta\left(a_{2 k+1}, a_{2 k+2}\right) \leq \alpha \frac{\vartheta\left(a_{2 k}, a_{2 k+1}\right)}{2(1-\beta)}+\alpha \frac{\vartheta\left(b_{2 k}, b_{2 k+1}\right)}{2(1-\beta)}$

Similarly

$\vartheta\left(b_{2 k+1}, b_{2 k+2}\right) \leq \alpha \frac{\vartheta\left(b_{2 k}, b_{2 k+1}\right)}{2(1-\beta)}+\alpha \frac{\vartheta\left(a_{2 k}, a_{2 k+1}\right)}{2(1-\beta)}$

\section{Common Coupled Fixed Point Theorems}

Add (3.2) and (3.3)

$\left[\vartheta\left(a_{2 k+1}, a_{2 k+2}\right)+\vartheta\left(b_{2 k+1}, b_{2 k+2}\right)\right]$

$$
\begin{aligned}
& \leq \frac{\alpha}{(1-\beta)}\left[\vartheta\left(a_{2 k}, a_{2 k+1}\right)\right. \\
& \left.+\vartheta\left(b_{2 k}, b_{2 k+1}\right)\right]
\end{aligned}
$$

$=h\left[\vartheta\left(a_{2 k}, a_{2 k+1}\right)+\vartheta\left(b_{2 k}, b_{2 k+1}\right)\right]$

Where $0<h=\frac{\alpha}{(1-\beta)}<1$. similarly

$\vartheta\left(a_{2 k+2}, a_{2 k+3}\right) \leq \alpha \frac{\vartheta\left(a_{2 k+1}, a_{2 k+2}\right)}{2(1-\beta)}+\alpha \frac{\vartheta\left(b_{2 k+1}, b_{2 k+2}\right.}{2(1-\beta)}$

Similarly

$\vartheta\left(b_{2 k+2}, b_{2 k+3}\right) \leq \alpha \frac{\vartheta\left(b_{2 k+1}, b_{2 k+2}\right)}{2(1-\beta)}+\alpha \frac{\vartheta\left(a_{2 k+1}, a_{2 k+2}\right)}{2(1-\beta)}$

Adding above equation, we get

$\left[\vartheta\left(a_{2 k+2}, a_{2 k+3}\right)+\vartheta\left(b_{2 k+2}, b_{2 k+3}\right)\right]$

$$
\begin{aligned}
& \leq \frac{\alpha}{(1-\beta)}\left[\vartheta\left(a_{2 k+1}, a_{2 k+2}\right)\right. \\
&\left.+\vartheta\left(b_{2 k+1}, b_{2 k+2}\right)\right] \\
&=h\left[\vartheta\left(a_{2 k+1}, a_{2 k+2}\right)+\vartheta\left(b_{2 k+1}, b_{2 k+2}\right)\right] \\
& \text { Continuing in this way, } \\
&\left(\vartheta\left(a_{n}, a_{n+1}\right)+\vartheta\right.\left.\left(b_{n}, b_{n+1}\right)\right) \\
& \leq h\left(\vartheta\left(a_{n-1}, a_{n}\right)+\vartheta\left(b_{n-1}, b_{n}\right) \leq---\right. \\
& \leq h^{n}\left(\vartheta\left(a_{0}, a_{1}\right)+\vartheta\left(b_{0}, b_{1}\right)\right.
\end{aligned}
$$

Now, if

$\vartheta\left(a_{n}, a_{n+1}\right)+\vartheta\left(b_{n}, b_{n+1}\right)=\delta_{n}$, then $\delta_{n}$, then $\delta_{n}$ $\leq h \delta_{n-1} \leq---\leq h^{n} \delta 0$

For $m>n$, we have

$$
\begin{aligned}
& \left(\vartheta\left(a_{n}, a_{m}\right)+\vartheta\left(b_{n}, b_{m}\right)\right) \leq K\left(\vartheta\left(a_{n}, a_{n+1}\right)+\right. \\
& \vartheta b n, b n+1+\ldots+K m-n(\vartheta a m-1, a m+\vartheta b m-1, b,) \\
& \leq K h^{n} \delta_{0}+K^{2} h^{n+1} \delta 0+\cdots+K^{m-n} h^{m-1} \delta_{0} \\
& <K h^{n}\left[1+(K h)+(K h)^{2}+\cdots\right] \delta_{0} \\
& =\frac{K h^{n}}{1-K h} \delta_{0} \rightarrow 0 \text { as } n \rightarrow \infty
\end{aligned}
$$

This shows that $\left\{a_{n}\right\}$ and $\left\{b_{n}\right\}$ are Cauchy sequence in $X$. Since $X$ is a complete b-metric-like space, there exists $a, b \in X$ such that $a_{n} \rightarrow \infty$ and $b_{n} \rightarrow \infty$.

Step 2 : Now, We show that $a=S(a, b)$ and $b=S(b, a)$.

We suppose on the contrary that $a \neq S(a, b)$ and $b \neq$ $S(b, a)$ so that

$\vartheta(a, S(a, b))=l_{1}>0$ and $\vartheta(b, S(b, a))=l_{2}>0$

Consider

$l_{1}=\vartheta(a, S(a, b)) \leq K\left[\vartheta\left(a, a_{2 k+2}\right)+\vartheta\left(a_{2 k+2}, S(a, b)\right)\right]$

$\leq K \vartheta\left(a, a_{2 k+2}\right)+K \vartheta\left(T\left(a_{2 k+1}, b_{2 k+1}\right), S(a, b)\right)$

$\leq K \vartheta\left(a, a_{2 k+2}\right)+K a \frac{\vartheta\left(a_{2 k+1}, a\right)+\vartheta\left(b_{2 k+1}, b\right)}{2}$

$+K \beta \frac{\vartheta(a, S(a, b)) \vartheta\left(a_{2 k+1}, T\left(a_{2 k+1}, b_{2 k+1}\right)\right)}{1+\vartheta\left(a_{2 k+1}, a\right)+\vartheta\left(b_{2 k+1}, b\right)}+$

$K \gamma \frac{\vartheta\left(a_{2 k+1}, S(a, b)\right) \vartheta\left(a, a_{2 k+2}\right)}{1+\vartheta\left(a_{2 k+1}, a\right)+\vartheta\left(b_{2 k+1, b}\right)}$

By taking $k \rightarrow \infty$, we get

$l_{1} \leq 0$, which is contradiction.

Therefore, $\vartheta(a, S(a, b))=0$. this implies $a=S(a, b)$.

Similarly, we can prove that $b=S(b, a)$.

It follows similarly we can show that $a=T(a, b)$ and $T(b, a)$.

So we have proved that $(a, b)$ is a common coupled fixed point of $S$ and $T$.

Step 3 : We now show that $S$ and $T$ have a unique common coupled fixed point.

Let $\left(a^{*}, b^{*}\right) \in X \times X$ be another common coupled fixed point of $S$ and $T$. Then

$\vartheta\left(a^{*}, a^{*}\right)=\vartheta\left(S(a, b), T\left(a^{*}, b^{*}\right)\right)$

$\leq \alpha \frac{\vartheta\left(a, a^{*}\right)+\vartheta\left(b, b^{*}\right)}{2}$

$$
\begin{aligned}
& +\beta \frac{\vartheta(a, S(a, b)) \vartheta\left(a^{*}, T\left(a^{*}, T\left(a^{*}, b^{*}\right)\right)\right.}{1+\vartheta\left(a, a^{*}\right)+\vartheta\left(b, b^{*}\right)} \\
& +\gamma \frac{\vartheta\left(a^{*}, S(a, b)\right) \vartheta\left(a, T\left(a^{*}, b^{*}\right)\right)}{1+\vartheta\left(a, a^{*}\right)+\vartheta\left(b, b^{*}\right)} \\
& =\alpha \frac{\vartheta\left(a, a^{*}\right)+\vartheta\left(b, b^{*}\right)}{2}+\beta \frac{\vartheta(a, a) \vartheta\left(a^{*}, a^{*}\right)}{1+\vartheta\left(a, a^{*}\right)+\vartheta\left(b, b^{*}\right)} \\
& +\gamma \frac{\vartheta\left(a^{*}, a\right) \vartheta\left(a, a^{*}\right)}{1+\vartheta\left(a, a^{*}\right)+\vartheta\left(b, b^{*}\right)} \\
& \vartheta\left(a, a^{*}\right) \leq \frac{\alpha}{2} \vartheta\left(a, a^{*}\right)+\frac{\alpha}{2} \vartheta\left(b, b^{*}\right)+4 \beta \vartheta\left(a, a^{*}\right) \\
& \vartheta\left(a, a^{*}\right) \leq \frac{+\gamma \vartheta\left(a^{*} a\right)}{(2-\alpha-8 \beta-2 \gamma)} \vartheta\left(b, b^{*}\right) \\
& \leq \frac{\alpha}{(2-\alpha-2 \gamma)} \vartheta\left(b, b^{*}\right)
\end{aligned}
$$

Similarly, we can easily prove that 


\section{International Journal of Science and Research (IJSR) \\ ISSN (Online): 2319-7064}

Index Copernicus Value (2013): 6.14 | Impact Factor (2014): 5.611

$\vartheta\left(b, b^{*}\right) \leq \frac{\alpha}{(2-\alpha-2 \gamma)} \vartheta\left(a, a^{*}\right)$

Adding, we get

$\vartheta\left(a, a^{*}\right)+\vartheta\left(b, b^{*}\right) \leq \frac{\alpha}{(2-\alpha-2 \gamma)}\left[\vartheta\left(a, a^{*}\right)+\vartheta\left(b, b^{*}\right)\right]$.

$(2-2 \alpha-2 \gamma)\left[\vartheta\left(a, a^{*}\right)+\vartheta\left(b, b^{*}\right)\right] \leq 0$

$\vartheta\left(a, a^{*}\right)+\vartheta\left(b, b^{*}\right)=0$.

This implies, $a=a^{*}$ and $b=b^{*}$

\section{References}

[1] Alghamdi, M.A, Hussain, N. and Salimi, P. Fixed point and coupled fixed point theorems on b-metric-like spaces. Journal of Inequalities and Applications 2013 2013:402.

[2] Amini-Harandi, A : Metric like spaces, partial metric spaces and fixed points. Fixed Point Theory and Applications 2012, 2012:204.

[3] Czerwik, S., Contraction mappings in b-metric spaces. Acta Mathematica et Informatica Universitatis Ostraviensis, 1(1), 1993, 5-11.

[4] Akkouchi, M., common fixed point theorem for expansive mappings under strict implicit conditions on bmetric space. Acta Univ. Palack Olomuc. Fac. Rerum Natur. Math., 50(2011), 5-15 